\title{
CO-REPRESENTATIONS OF HOPF-VON NEUMANN ALGEBRAS ON OPERATOR SPACES OTHER THAN COLUMN HILBERT SPACE
}

\author{
VOLKER RUNDE
}

(Received 21 October 2009)

\begin{abstract}
Recently, Daws introduced a notion of co-representation of abelian Hopf-von Neumann algebras on general reflexive Banach spaces. In this note, we show that this notion cannot be extended beyond subhomogeneous Hopf-von Neumann algebras. The key is our observation that, for a von Neumann algebra $\mathfrak{M}$ and a reflexive operator space $E$, the normal spatial tensor product $\mathfrak{M} \bar{\otimes} \mathcal{C} B(E)$ is a Banach algebra if and only if $\mathfrak{M}$ is subhomogeneous or $E$ is completely isomorphic to column Hilbert space.
\end{abstract}

2000 Mathematics subject classification: primary 47L30; secondary 16T10, 46L99, 47L25.

Keywords and phrases: Hopf-von Neumann algebra, co-representation, reflexive operator space, operator algebra, column Hilbert space.

\section{Introduction}

If $\mathfrak{A}$ is a Banach algebra, then its dual space $\mathfrak{A}^{*}$ is a Banach $\mathfrak{A}$-bimodule through

$$
\langle x, a \cdot \phi\rangle:=\langle x a, \phi\rangle \quad \text { and } \quad\langle x, \phi \cdot a\rangle:=\langle a x, \phi\rangle \quad\left(a, x \in \mathfrak{A}, \phi \in \mathfrak{A}^{*}\right) .
$$

A functional $\phi \in \mathfrak{A}^{*}$ is said to be weakly almost periodic if $\{a \cdot \phi: a \in \mathfrak{A},\|a\| \leq 1\}$ is relatively weakly compact in $\mathfrak{A}^{*}$. There appears to be some asymmetry in the definition of a weakly almost periodic functional, but thanks to Grothendieck's double limit criterion [7, Proposition 4], $\phi \in \mathfrak{A}^{*}$ is weakly almost periodic if and only if $\{\phi \cdot a: a \in \mathfrak{A},\|a\| \leq 1\}$ is relatively weakly compact in $\mathfrak{A}^{*}$. The collection of all weakly almost periodic functionals on $\mathfrak{A}$ is a closed subspace of $\mathfrak{A}^{*}$, which we denote by WAP( $\mathfrak{A})$.

Let $G$ be a locally compact group, and let $\mathfrak{A}$ be its group algebra $L^{1}(G)$. In this case, it is not difficult to see that $\operatorname{WAP}(\mathfrak{A})$ is nothing more than $\operatorname{WAP}(G)$, the commutative $C^{*}$-algebra of all weakly almost periodic functions on $G$ (see [3] for the definition and properties of WAP $(G)$ ). Now, let $\mathfrak{A}$ be Eymard's Fourier algebra $A(G)$ with dual $\operatorname{VN}(G)$ (see [6]); in this case, we denote $\operatorname{WAP}(\mathfrak{A})$ by $\operatorname{WAP}(\hat{G})$. If $G$ is abelian, then $\operatorname{WAP}(\hat{G})$ is indeed just the weakly almost periodic functions on the dual group $\hat{G}$

(C) 2010 Australian Mathematical Publishing Association Inc. 0004-9727/2010 \$16.00 
and therefore, in particular, is a $C^{*}$-subalgebra of $L^{\infty}(\hat{G}) \cong \mathrm{VN}(G)$. With a little more effort, one can show that $\operatorname{WAP}(\hat{G})$ is a $C^{*}$-subalgebra of $\operatorname{VN}(G)$ whenever $G$ has an abelian subgroup of finite index. For general $G$, however, it has been an open question for decades whether or not $\operatorname{WAP}(\hat{G})$ is always a $C^{*}$-subalgebra of $\operatorname{VN}(G)$.

\section{Hopf-von Neumann algebras and co-representations}

Recently, Daws showed in [4] that WAP $(M(G))$ is a $C^{*}$-subalgebra of $\mathcal{C}_{0}(G)^{* *}$ for any $G$, where $M(G)$ is the measure algebra of $G$. In fact, Daws proved a much more general result about abelian Hopf-von Neumann algebras.

Definition 2.1. A Hopf-von Neumann algebra is a pair $(\mathfrak{M}, \Gamma)$, where $\mathfrak{M}$ is a von Neumann algebra, and $\Gamma$ is a co-multiplication: a unital, injective, normal *-homomorphism $\Gamma: \mathfrak{M} \rightarrow \mathfrak{M} \bar{\otimes} \mathfrak{M}$ which is co-associative, that is,

$$
(\mathrm{id} \otimes \Gamma) \circ \Gamma=(\Gamma \otimes \mathrm{id}) \circ \Gamma .
$$

We call a Hopf-von Neumann algebra $(\mathfrak{M}, \Gamma)$ abelian, semidiscrete, and so on, if the underlying von Neumann algebra $\mathfrak{M}$ has the corresponding property.

EXAMPLE 2.2. Let $G$ be a locally compact group.

(a) A co-multiplication

$$
\Gamma: L^{\infty}(G) \rightarrow L^{\infty}(G) \bar{\otimes} L^{\infty}(G) \cong L^{\infty}(G \times G)
$$

is given by

$$
(\Gamma \phi)(x, y):=\phi(x y) \quad\left(\phi \in L^{\infty}(G), x, y \in G\right) .
$$

(Restricting $\Gamma$ to $\mathcal{C}_{0}(G)$ and then taking section adjoints yields another comultiplication $\tilde{\Gamma}: \mathcal{C}_{0}(G)^{* *} \rightarrow \mathcal{C}_{0}(G)^{* *} \bar{\otimes} \mathcal{C}_{0}(G)^{* *}$.)

(b) Let $\lambda: G \rightarrow \mathcal{B}\left(L^{2}(G)\right)$ be the left regular representation of $G$. Then a comultiplication $\hat{\Gamma}: \mathrm{VN}(G) \rightarrow \mathrm{VN}(G) \bar{\otimes} \mathrm{VN}(G)$ is given by

$$
\hat{\Gamma}(\lambda(x))=\lambda(x) \otimes \lambda(x) \quad(x \in G) .
$$

Whenever $(\mathfrak{M}, \Gamma)$ is a Hopf-von Neumann algebra, the unique predual $\mathfrak{M}_{*}$ of $\mathfrak{M}$ becomes a Banach algebra via

$$
\langle x, f * g\rangle:=\langle\Gamma x, f \otimes g\rangle \quad\left(f, g \in \mathfrak{M}_{*}, x \in \mathfrak{M}\right) .
$$

EXAMPLE 2.3. If $G$ is a locally compact group, then $*$ defined in this manner for $\left(L^{\infty}(G), \Gamma\right)$ is the usual convolution product on $L^{1}(G)$, whereas $*$ for $(\operatorname{VN}(G), \hat{\Gamma})$ is the pointwise product on $A(G)$.

Any von Neumann algebra $\mathfrak{M}$ is a (concrete) operator space, so that $\mathfrak{M}_{*}$ is an abstract operator space. (For background on operator space theory, we refer to [5], the notation of which we adopt.) If $(\mathfrak{M}, \Gamma)$ is a Hopf-von Neumann algebra, then $\Gamma$ is a complete isometry. Consequently, $\left(\mathfrak{M}_{*}, *\right)$ is not only a Banach algebra, but a completely contractive Banach algebra [5, p. 308]. 
The main result of [4] is as follows.

THEOREM 2.4. Let $(\mathfrak{M}, \Gamma)$ be an abelian Hopf-von Neumann algebra. Then $\mathrm{WAP}\left(\mathfrak{M}_{*}\right)$ is a $C^{*}$-subalgebra of $\mathfrak{M}$.

At the heart of Daws's proof is the notion of a co-representation of a Hopf-von Neumann algebra. Usually one considers co-representation on Hilbert spaces.

Definition 2.5. Let ( $\mathfrak{M}, \Gamma)$ be a Hopf-von Neumann algebra. A co-representation of $(\mathfrak{M}, \Gamma)$ on a Hilbert space $\mathfrak{H}$ is an operator $U \in \mathfrak{M} \bar{\otimes} \mathcal{B}(\mathfrak{H})$ such that

$$
(\Gamma \otimes \mathrm{id})(U)=U_{1,3} U_{2,3} \text {. }
$$

Here, $U_{1,3}$ and $U_{2,3}$ are what is called leg notation: if $\mathfrak{M}$ acts on a Hilbert space $\mathfrak{K}$, then $U_{1,3}$ is the linear operator on the Hilbert space tensor product $\mathfrak{K} \otimes_{2} \mathfrak{K} \otimes_{2} \mathfrak{H}$ that acts as $U$ on the first and the third factor and as the identity on the second one; $U_{2,3}$ is defined similarly.

Commonly, co-representations are also required to be unitary, but we will not need that property.

By [5, Corollary 7.1.5 and Theorem 7.2.4], we have the completely isometric identifications

$$
\mathfrak{M} \bar{\otimes} \mathcal{B}(\mathfrak{H})=\left(\mathfrak{M}_{*} \hat{\otimes} \mathcal{B}(\mathfrak{H})_{*}\right)^{*}=\mathcal{C} B\left(\mathfrak{M}_{*}, \mathcal{B}(\mathfrak{H})\right) .
$$

Given an operator $U \in \mathfrak{M} \otimes \mathcal{B}(\mathfrak{H})$, the corresponding map in $\mathcal{C} B\left(\mathfrak{M}_{*}, \mathcal{B}(\mathfrak{H})\right)$ is

$$
\mathfrak{M}_{*} \rightarrow \mathcal{B}(\mathfrak{H}), \quad f \mapsto(f \otimes \mathrm{id})(U),
$$

and $(*)$ is equivalent to $(* *)$ being a multiplicative map from $\left(\mathfrak{M}_{*}, *\right)$ into $\mathcal{B}(\mathfrak{H})$. The advantage of looking at elements of $\mathfrak{M} \otimes \mathcal{B}(\mathfrak{H})$ instead of $\mathcal{C} B\left(\mathfrak{M}_{*}, \mathcal{B}(\mathfrak{H})\right)$ is that $\mathfrak{M} \otimes \mathcal{B}(\mathfrak{H})$ is again a von Neumann algebra, so that it makes sense to multiply its elements.

Let $(\mathfrak{M}, \Gamma)$ be an abelian Hopf-von Neumann algebra. Then $\mathfrak{M}$ is of the form $L^{\infty}(X)$ for some measure space $X$. The proof of Theorem 2.4 in [4] has three main ingredients.

(1) Elements of WAP $\left(L^{1}(X)\right)$ arise as coefficients of representations of $\left(L^{1}(X), *\right)$ on reflexive Banach spaces.

(2) For a reflexive Banach space $E$, the weak* closure of $L^{\infty}(X) \otimes \mathcal{B}(E)$ in $\mathcal{B}\left(L^{2}(X, E)\right)$, denoted by $L^{\infty}(X) \bar{\otimes} \mathcal{B}(E)$, can be identified with $\mathcal{B}\left(L^{1}(X)\right.$, $\mathcal{B}(E)$ ) [4, Proposition 3.2]. This identification then allows co-representations of $\left(L^{\infty}(X), \Gamma\right)$ to be defined on $E$ by analogy with Definition 2.5.

(3) The product in $L^{\infty}(X) \bar{\otimes} \mathcal{B}(E)$ corresponds to the product in $\operatorname{WAP}\left(L^{1}(X)\right)$.

Is it possible to adapt this approach to more general Hopf-von Neumann algebras?

\section{Co-representations on operator spaces}

In [4], Daws uses the symbol $L^{\infty}(X) \bar{\otimes} \mathcal{B}(E)$ for the closure of $L^{\infty}(X) \otimes \mathcal{B}(E)$ in $\mathcal{B}\left(L^{2}(X, E)\right)$. In operator space theory, the symbol $\bar{\otimes}$ is usually reserved for the 
normal spatial tensor product of dual operator spaces [5, p. 134]. Both usages are consistent: for a reflexive Banach space $E$, we have the isometric identifications

$$
\begin{aligned}
\mathcal{B}\left(L^{1}(X), \mathcal{B}(E)\right) & =\mathcal{C} B\left(L^{1}(X), \mathcal{C} B(\max E)\right) \\
& =\left(L^{1}(X) \hat{\otimes}\left(\max E \hat{\otimes} \min E^{*}\right)\right)^{*} \\
& =L^{\infty}(X) \bar{\otimes} \mathcal{C} B(\max E) .
\end{aligned}
$$

From [4, Proposition 3.2], we thus conclude that the product on $L^{\infty}(X) \otimes \mathcal{B}(E)$ extends to $L^{\infty}(X) \bar{\otimes} \mathcal{C} B(\max E)$, turning it into a Banach algebra. More generally, $L^{\infty}(X) \bar{\otimes} \mathcal{C} B(E)$ is a Banach algebra for any reflexive, homogeneous operator space $E$, that is, satisfying $\mathcal{C} B(E)=\mathcal{B}(E)$ with identical norms.

Let $\mathfrak{M}$ be a semidiscrete von Neumann algebra, and let $E$ be a reflexive operator space. Then we have the completely isometric identifications

$$
\mathcal{C} B\left(\mathfrak{M}_{*}, \mathcal{C} B(E)\right)=\left(\mathfrak{M}_{*} \hat{\otimes}\left(E \hat{\otimes} E^{*}\right)\right)^{*}=\mathfrak{M} \bar{\otimes} \mathcal{C} B(E) .
$$

(We need the semidiscreteness of $\mathfrak{M}$ for the second equality: without it, we would not get $\mathfrak{M} \bar{\otimes} \mathcal{C} B(E)$, but the normal Fubini tensor product $\mathfrak{M} \bar{\otimes}_{\mathcal{F}} \mathcal{C} B(E)$; see [8].) This suggests that it might be possible to define a notion of co-representation of semidiscrete Hopf-von Neumann algebras on general reflexive operator spaces. Just to meaningfully state the right-hand side (*) for some $U \in \mathfrak{M} \bar{\otimes} \mathcal{C} B(E)$, we need $\mathfrak{M} \bar{\otimes} \mathfrak{M} \bar{\otimes} \mathcal{C} B(E)$ to be multiplicatively closed, and to adapt the proof of Theorem 2.4 to general (semidiscrete) Hopf-von Neumann algebras, we need $\mathfrak{M} \bar{\otimes} B(E)$ also to be multiplicatively closed. It all comes down to the question whether or not $\mathfrak{M} \bar{\otimes} \mathcal{C} B(E)$ is a Banach algebra for a (semidiscrete) von Neumann algebra and a reflexive operator space $E$.

For abelian $(\mathfrak{M}, \Gamma)$, this is indeed the case, and it is not difficult to extend [4, Proposition 3.2] to a general operator space setting.

Proposition 3.1. Let $L^{\infty}(X)$ be an abelian von Neumann algebra, and let $E$ be a reflexive operator space. Then the closure of $L^{\infty}(X) \otimes \mathcal{C} B(E)$ in $\mathcal{C} B\left(L^{2}(X, E)\right)$ is isometrically isomorphic to $\mathcal{C} B\left(L^{1}(X), \mathcal{C} B(E)\right)$. In particular, the product of $L^{\infty}(X) \otimes \mathcal{C} B(E)$ extends to $L^{\infty}(X) \bar{\otimes} \mathcal{C} B(E)$, turning it into a Banach algebra.

Here, the operator space structure on $L^{2}(X, E)$ is that obtained through complex interpolation between $L^{\infty}(X) \ddot{\otimes} E$ and $L^{1}(X) \hat{\otimes} E$ as described in [9].

We can even go a little beyond the abelian framework. If $\mathfrak{M}$ is a subhomogeneous von Neumann algebra, it is of the form

$$
\mathfrak{M} \cong M_{n_{1}}\left(\mathfrak{M}_{1}\right) \oplus \cdots \oplus M_{n_{k}}\left(\mathfrak{M}_{k}\right)
$$

with $n_{1}, \ldots, n_{k} \in \mathbb{N}$ and abelian von Neumann algebras $\mathfrak{M}_{1}, \ldots, \mathfrak{M}_{k}$. This yields the following corollary.

COROLlary 3.2. Let $\mathfrak{M}$ be a subhomogeneous von Neumann algebra, and let $E$ be a reflexive operator space. Then the product of $\mathfrak{M} \otimes \mathcal{C} B(E)$ extends to $\mathfrak{M} \bar{\otimes} B(E)$, 
turning it into a Banach algebra (with bounded, but not necessarily contractive multiplication).

So, if $(\mathfrak{M}, \Gamma)$ is a subhomogeneous Hopf-von Neumann algebra, we can meaningfully speak of its co-representations on reflexive operator spaces. But what if we go beyond subhomogeneous von Neumann algebras? For certain operator spaces, this is no problem. Let $\mathfrak{H}$ be a Hilbert space, and let $\mathfrak{H}_{c}$ be column Hilbert space [5, Section 3.4]. Then $\mathcal{C} B\left(\mathfrak{H}_{c}\right)=\mathcal{B}(\mathfrak{H})$ as operator spaces [5, Theorem 3.4.1], so that

$$
\mathfrak{M} \bar{\otimes} \mathcal{C} B\left(\mathfrak{H}_{c}\right)=\mathfrak{M} \bar{\otimes} \mathcal{B}(\mathfrak{H})
$$

is a von Neumann algebra, and co-representations on $\mathfrak{H}_{c}$ are nothing more than corepresentations on $\mathfrak{H}$ in the sense of Definition 2.5.

As we shall see, this is about as far as we can get, and we state the following theorem.

THEOREM 3.3. Let (M, $\Gamma)$ be a Hopf-von Neumann algebra, and let $E$ be a reflexive operator space. Then the following are equivalent.

(i) The product of $\mathfrak{M} \otimes \mathcal{C} B(E)$ extends to $\mathfrak{M} \bar{\otimes} B(E)$, turning it into a Banach algebra with bounded, but not necessarily contractive multiplication.

(ii) The product of $\mathfrak{M} \otimes \mathcal{C} B(E)$ extends to $\mathfrak{M} \otimes \check{C} B(E)$, turning it into a Banach algebra with bounded, but not necessarily contractive multiplication.

(iii) $\mathfrak{M}$ is subhomogeneous or $E$ is completely isomorphic to $\mathfrak{H}_{c}$ for some Hilbert space $\mathfrak{H}$.

For the proof, we require a lemma.

LEMMA 3.4. Let $\mathfrak{A}$ and $\mathfrak{B}$ be completely contractive Banach algebras such that $\mathfrak{A}$ contains the full matrix algebra $M_{n}$ as a subalgebra for each $n \in \mathbb{N}$, and suppose that the product of $\mathfrak{A} \otimes \mathfrak{B}$ extends to $\mathfrak{A} \check{\otimes} \mathfrak{B}$, turning it into a Banach algebra with bounded, but not necessarily contractive multiplication. Then $\mathfrak{B}$ is completely isomorphic to an operator algebra.

Here, an operator algebra is a closed, but not necessarily self-adjoint subalgebra of $\mathcal{B}(\mathfrak{H})$ for some Hilbert space $\mathfrak{H}$.

PROOF. Let $C \geq 1$ be a bound for the multiplication in $\mathfrak{A} \check{\otimes} \mathfrak{B}$, and note that, for $n \in$ $\mathbb{N}$, we have a complete isometry $M_{n}(\mathfrak{B}) \cong M_{n} \check{\otimes} \mathfrak{B}$ [5, Corollary 8.1.3]. Since $M_{n}$ is a subalgebra of $\mathfrak{A}$, this means that formal matrix multiplication from $M_{n}(\mathfrak{B}) \times M_{n}(\mathfrak{B})$ to $M_{n}(\mathfrak{B})$ is bounded by $C$ for each $n$. By the definition of the Haagerup tensor product (see [5, Ch. 9]) $\mathfrak{B} \otimes^{h} \mathfrak{B}$, this means that multiplication $m: \mathfrak{B} \otimes \mathfrak{B} \rightarrow \mathfrak{B}$ extends to a completely bounded map $m: \mathfrak{B} \otimes^{h} \mathfrak{B} \rightarrow \mathfrak{B}$. Hence, $\mathfrak{B}$ is completely isomorphic to an operator algebra by [2, Theorem 5.2.1].

Proof of TheOREM 3.3. We have previously seen that (iii) $\Longrightarrow$ (i) holds, and (i) $\Longrightarrow$ (ii) is obvious.

To prove (ii) $\Longrightarrow$ (iii), assume that $\mathfrak{M}$ is not subhomogeneous. Then the structure theory of von Neumann algebras yields that $\mathfrak{M}$ contains $M_{n}$ as a subalgebra for each $n$. 
By the lemma, $\mathcal{C} B(E)$ is thus completely isomorphic to an operator algebra. By [1, Theorem 3.4], this means that $E$ is completely isomorphic to $\mathfrak{H}_{c}$ for some Hilbert space $\mathfrak{H}$.

\section{References}

[1] D. P. Blecher, 'A completely bounded characterization of operator algebras', Math. Ann. 303 (1995), 227-239.

[2] D. P. Blecher and C. Le Merdy, Operator Algebras and their Modules. An Operator Space Approach, London Mathematical Society Monographs (New Series), 30 (Clarendon Press, Oxford, 2004).

[3] R. B. Burckel, Weakly Almost Periodic Functions on Semigroups (Gordon and Breach Science Publishers, New York, 1970).

[4] M. Daws, 'Weakly almost periodic functionals on the measure algebra', Math. Z. 265 (2010), 61-78.

[5] E. G. Effros and Z.-J. Ruan, Operator Spaces, London Mathematical Society Monographs (New Series), 23 (Clarendon Press, Oxford, 2000).

[6] P. Eymard, 'L'algèbre de Fourier d'un groupe localement compact', Bull. Soc. Math. France 92 (1964), 181-236.

[7] A. Grothendieck, 'Critères de compacité dans les espaces fonctionnels généraux', Amer. J. Math. 74 (1953), 168-186.

[8] J. Kraus, 'The slice map problem for $\sigma$-weakly closed subspaces of von Neumann algebras', Trans. Amer. Math. Soc. 279 (1983), 357-376.

[9] G. Pisier, 'Non-commutative vector valued $L_{p}$-spaces and completely $p$-summing maps', Astérisque 247 (1998).

VOLKER RUNDE, Department of Mathematical and Statistical Sciences,

University of Alberta, Edmonton, Alberta, Canada T6G 2G1

e-mail: vrunde@ualberta.ca 